\title{
In Situ Liquid-Phase-Adsorption Measurement System Based on Fiber-Optic Sensing with the Aid of Membranes
}

\author{
Fei Gao, ${ }^{\dagger}$ Turghun Muhammad, ${ }^{* \dagger}{ }^{\dagger}$ Mahinur Bakri, ${ }^{\ddagger}$ Parezhati Pataer, ${ }^{\dagger}$ and Lingxin Chen $^{\S}$ (0)
}

${ }^{\dagger}$ Key Laboratory of Oil and Gas Fine Chemicals, Ministry of Education \& Xinjiang Uygur Autonomous Region, College of Chemistry and Chemical Engineering, Xinjiang University, Urumqi 830046, China

${ }^{\ddagger}$ The Key Laboratory of Plant Resources and Chemistry of Arid Zone, Xinjiang Technical Institute of Physics and Chemistry, Chinese Academy of Sciences, Urumqi 8300, China

${ }^{\S}$ Key Laboratory of Coastal Environment Processes and Ecological Remediation, Yantai Institute of Coastal Zone Research, Chinese Academy of Sciences, Yantai 264003, China

Supporting Information

ABSTRACT: At present, liquid phase adsorption (LPA) is still being quantitatively characterized in the way of manual sampling and off-line determination because of the complexity of the system comparing to gas adsorption. This paper describes a novel method for in situ, real-time measurement of LPA in general based on fiber-optic sensing (FOS) with the aid of membranes for the first time. A self-made measurement vessel was assembled from an adsorption bag, thermostatic devices with a stirrer, and a fiber-optic dipping probe. Also, macroporous adsorption resins (MARs) and rutin were chosen as model adsorbent and adsorbate to establish the FOS system. Here, in situ light absorption measurement was

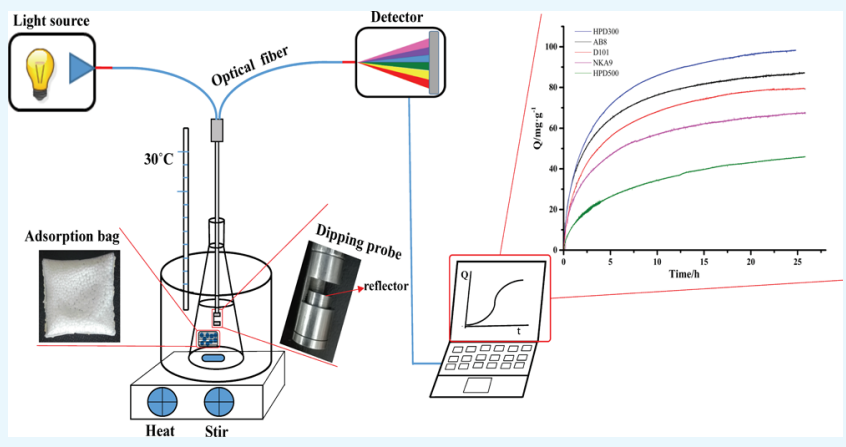
achieved by eliminating interference of adsorbent particles via encapsulating them with a membrane into the adsorption bag. In situ LPA measurement of rutin solution on MARs was obtained by detecting light absorption at $353 \mathrm{~nm}$ using dipping probe, in the broad concentration range from 0.3 to $60 \mathrm{mg} / \mathrm{L}$ with excellent linearity $\left(R^{2}=0.9996\right)$. In situ measurements of adsorption and desorption kinetics on five kinds of MARs with different polarities were systematically carried out, showing that the adsorption process obeyed the pseudo-second-model. As well as, the system was proved to be highly accurate and reproducible. More importantly, this method enabled to study the initial stage of the adsorption process, starting from the time of the first second, which is the most important part in the adsorption kinetics, and this is impossible for traditional sampling methods. The successful application of FOS to in situ measurement of LPA not only contributes to fast, automatic, and real-time monitoring of LPA process but also enriches the research connotation of adsorption.

\section{INTRODUCTION}

Adsorption is one of the most common phenomenon in nature. ${ }^{1}$ It is a surface phenomenon and is defined as the increase in concentration of a particular component at the surface or interface between two phases. ${ }^{2}$ The theory of gas phase adsorption has been developed relatively early and grown rapidly to maturity. The real-time in situ kinetic measurements were assembled for the first time by Thomas in 1991. ${ }^{3}$ In situ gas-phase thermal desorption spectroscopy to investigate the adsorption/desorption of hydrogen was reported by Takenouchi in $2015 .{ }^{4}$ In contrast, liquid phase adsorption (LPA) has been used in much wider areas, such as environmental remediation, ${ }^{5}$ purification of pharmaceuticals, ${ }^{6,7}$ and separation and purification of some components from the aqueous solution. ${ }^{8,9}$ However, nowadays LPA is still being investigated in a traditional way of manual sampling coupled with off-line determination.

Conventionally, the measurement of LPA includes several steps as follows: ${ }^{10}$ (i) adsorbents with certain weights are placed into a vessel, usually a conical flask, and a adsorbate solution with a given volume and concentration is added; (ii) after sealing the vessel, it is mounted in a thermostatic bath with agitation; (iii) after some time, a portion of the solution mixture is withdrawn from the vessel, centrifuged, or/and filtered; and (iv) finally, adsorption is analyzed by analytical techniques such as spectrophotometry or chromatography. Although so sophisticated modern analytical techniques such as $\mathrm{LC}-\mathrm{MS} / \mathrm{MS}^{11,12}$ and $\mathrm{GC}-\mathrm{MS}^{13}$ have been applied in the adsorption measurement, there are still many challenging issues.

The traditional procedure usually suffers from error and labor consumption due to manual sampling as well as from very limited data because frequent sampling easily causes big change to the adsorption system in terms of solution volume,

Received: May 18, 2018

Accepted: August 28, 2018

Published: September 10, 2018 
adsorbent amount, and the ratio between the two. Another issue challenging to conventional methods is the broad distribution of adsorption time. Some adsorptions, for example, methyl blue on boron nitride fibrous networks ${ }^{14}$ and benzotriazoles on zeolitic imidazolate framework- $8,{ }^{15}$ have fast rate, which only need $1 \mathrm{~min}$ to reach more than $50 \%$ adsorption and $5 \mathrm{~min}$ to equilibrium, respectively; some, on the other hand, have very slow adsorption rate, for example, rutin on HPD850 costing more than $70 \mathrm{~h}$ to approach equilibrium. $^{15,16}$ Continuous monitoring of solution to investigate the adsorption kinetics was achieved using a circulating pump and an interferometer, ${ }^{10}$ but the device is quite complex and not suitable for common adsorption systems. Adsorption of 4-nitrophenol on chitosan-based molecularly imprinted membrane was measured in situ in the cuvette by Di Bello in 2017, ${ }^{17}$ but this method is only suitable for the systems which have sufficient optically transparent window and for a volume of no more than few milliliters. In order to reduce the measurement procedure, some scholars applied adsorption bag made by nylon mesh and other materials in the batch adsorption and achieved direct determination of solution concentration without centrifugation or filtration at equilibrium. ${ }^{6,18,19}$ However, they still needed sampling of the solution. The more the volume and the number of sampling, the greater effect on the adsorption system and the more significant error were caused. Therefore, in situ, automatic and fast responsive analytical instrumentations/techniques are urgently required to be developed for LPA measurement.

Fiber-optic sensing (FOS) technology has become more mature and popular because of acceptable costs, compact instrumentation, high accuracy, and the capability of performing measurements in situ and real time, after over 30 years of innovation. ${ }^{20}$ As is well known, fiber-optics enable direct measurement of optical spectroscopy, from the infrared to the ultraviolet, in absorption, emission, and plasmonic resonance, to be performed at inaccessible sites, over large distances, in strong magnetic fields, and in harsh environments. ${ }^{21,22}$ Among the spectroscopy, the FOS based on UV-vis absorption has gained the most wide application owing to its low cost, simplicity, and high precision, typically in drug dissolution testing. $^{20,23-25}$ For the first time, Josefson reported a dissolution testing method, using felodipine tablet as an example, by means of optical fiber absorption spectrophotometer in turbid solution. ${ }^{26}$ Using this method, it is enabled to achieve in situ, real-time, and complete process measurement of tablet dissolution. Since then, optical fiber absorption spectrophotometer has been widely used alone ${ }^{20}$ and along with other techniques in highly diversified fields such as in situ study of catalytic kinetics, energy material characterization, explosive detection, ${ }^{27-29}$ and on-line sample preparation. ${ }^{30,31}$

Therefore, we purposed to present a novel method for in situ, real-time measurement of LPA based on FOS with UVvis absorption. The key component of the self-made FOS system was an adsorption vessel, which contained a conical flask, magnetic stirrer, fiber-optic probe, and adsorption bag. Macroporous adsorption resin (MAR) adsorbent and rutin (chemical structure shown in Figure S1) adsorbate were chosen as study models because they have been much investigated and well established. ${ }^{22,23,32,33}$ Also, the aspects of adsorption behavior, stability, reproducibility, and precision of the FOS system, as well as related comparison with traditional methods, were investigated in detail. To the best of our knowledge, this is the first report on in situ, automatic measurement of LPA for general sorbents, showing simplicity, high compatibility, and high accuracy.

\section{RESULTS AND DISCUSSION}

2.1. Quantitative Determination of Rutin Solution Using the FOS System. As shown in Figure 1, excellent

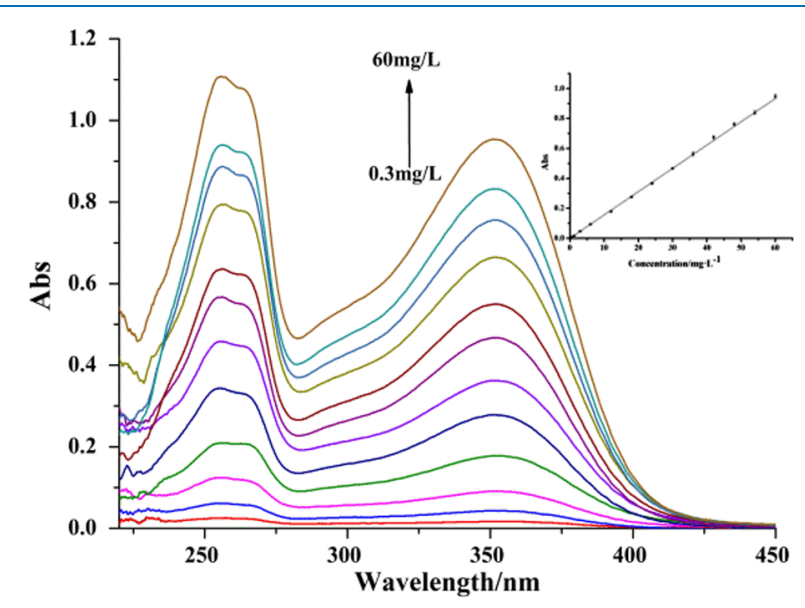

Figure 1. Absorption spectra and calibration curve of rutin aqueous solutions, the detection and reference wavelength were 353 and 453 $\mathrm{nm}$, respectively.

linearity at $353 \mathrm{~nm}$ over the range of $0.3-60 \mathrm{mg} / \mathrm{L}$ was attained, showing the regression equation of $A=0.016 \mathrm{C}+$ $0.002\left(R^{2}=0.9996\right)$, where $A$ relates to the absorbance of rutin and $C$ corresponds to the concentration of rutin $(\mathrm{mg} / \mathrm{L})$. The result suggested that in situ measurement of LPA could be realized by quantitative determination of rutin solution by using the self-made FOS system.

2.2. Effect of Membranes. In this study, we utilized large pore nylon net for making the adsorption bag. To evaluate the effect of nylon net on the adsorption of the MARs, batch adsorption was carried out with an empty bag as shown in Figure S2, and the nylon net did not adsorb rutin at all, indicating that its effect can be neglected. As for the effect of nylon net on the mass transfer in the form of adsorption bag, it was also demonstrated to be ignored. The effect of adsorption bag can be further minimized by optimization of the material and pore of the net bag.

2.3. Accuracy and Stability of the FOS System. In this experiment, the variance analysis of adsorption capacity was adopted to test the accuracy and stability of the in situ adsorption system. The adsorption kinetic curves of rutin on $\mathrm{AB}-8$ at $30{ }^{\circ} \mathrm{C}$ were obtained from six parallel experiments, as shown in the Figure S3, and the variance of adsorption capacity at every point were calculated by software program, Origin 9.0, as shown in the Figure S4.

The variance was found to be in the range of 0.82 and $2.55 \%$ for repetitive experiments tested by the FOS system. Also, there is no significant difference, indicating that the system can be well applied to in situ measurement of adsorption kinetics in batch adsorption/desorption with good stability, reliable data, and simple instrumentation.

2.4. Comparison of the Traditional Method. The need for a fast, accurate, and in situ detection has motivated us to develop an automatic, simple, accurate, high-precision, and wide-application method for LPA measurement. The out- 
standing feature of the optical-fiber-sensing LPA is that the whole process of adsorption is monitored in situ and real-time.

The adsorption capacities of rutin on resins were analyzed by the in situ LPA method and the traditional method, respectively, as shown in Figures 2 and S5. As seen, the

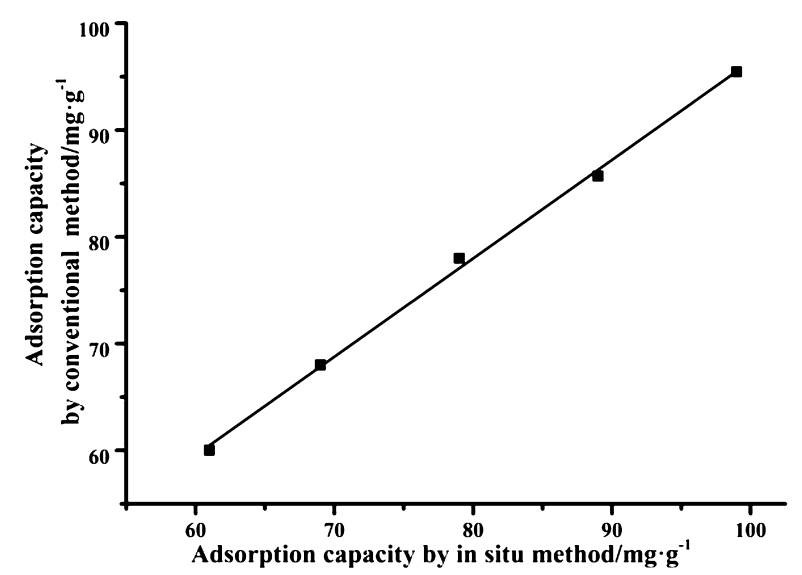

Figure 2. Comparison of adsorption capacity from the in situ method and the conventional method for rutin on different MARs (HPD500, NKA-9, D101, AB-8, HPD300), $C_{0}=60 \mathrm{mg} / \mathrm{L}, T=20^{\circ} \mathrm{C}$.

regression equation of in situ adsorption method versus traditional theoretical method was $y=0.9216 x+4.254\left(R^{2}=\right.$ 0.9975). It can be found that there was a good linear relationship between values obtained from the two methods. The slope and intercept of the line were satisfactorily close to the ideal values. ${ }^{34,35}$ Statistical analysis of the results shows that at the $95 \%$ level of confidence, the calculated $t$ value and $F$ value (Table S2) indicated that the proposed method was as precise and accurate as the conventional method. These results indicated that the in situ LPA method is in good agreement with the traditional method.

2.5. Adsorption Kinetic Curves on the Resins. Adsorption kinetics is one of the most important characteristics, which is responsible for the efficiency of sorption. During adsorption, the absorbance-time curve and the adsorption curves of rutin on resins (AB-8, D101, HPD300, HPD500, NKA-9) of different polarity are shown in Figure 3. It is clear that the adsorption capacity of rutin increased rapidly at the early stage (up to $75 \%$ uptake); the possible reason is that the diffusion rate of rutin to the macroporous pore is very fast because of plenty of binding sites and very high concentration of adsorbate available at this stage. Then, the adsorption rate slowed down gradually until it reached an equilibrium, after which there was no significant increase in rutin adsorption. It was also found that the adsorption rate and the capacity of rutin on nonpolar (HPD300, D101) and weakly polar (AB-8) resins are much higher than that of other resins, as shown in Figure 4. This means that functional groups of resin had a primary effect on rutin adsorption.

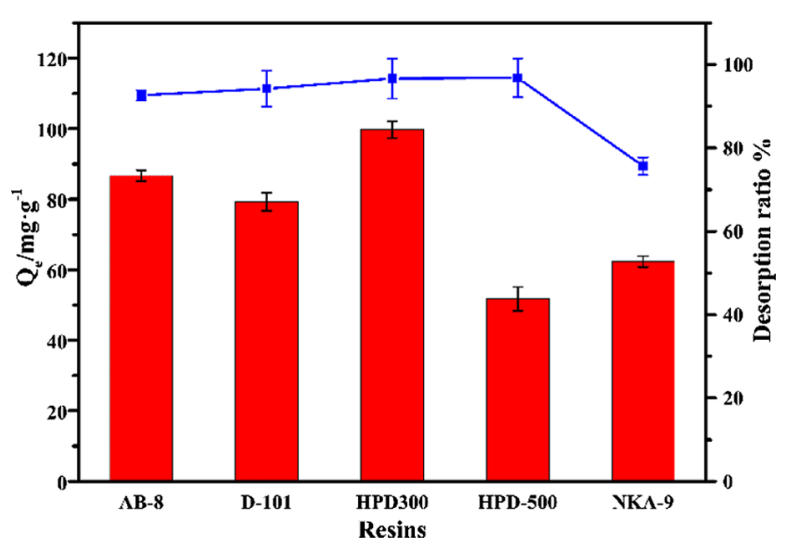

Figure 4. Adsorption capacity (red bars) and desorption ratio (blue line) of rutin on different MARs in aqueous solution, $C_{0}=60 \mathrm{mg} / \mathrm{L}, n$ $=3$.

2.6. Desorption Kinetic Curves. Ethanol aqueous solution ( $\mathrm{v} / \mathrm{v}=70 \%: 30 \%)$ was used as the desorption solvent, and the corresponding in situ desorption curves are shown in Figure 5a,b. It was obvious that rutin can be desorbed rapidly at the first $15 \mathrm{~min}$ and can be completely desorbed from all the tested resins. The desorption ratio was higher than $75 \%$ in the cases of all five resins, shown in Figure 4. The result of adsorption and desorption processes indicate that the system can be used for rapid detection of in situ adsorption and desorption measurement. Also, the resins can be used for the adsorption of flavonoids and easy to regenerate.

2.7. Adsorption Kinetic Models of Rutin on Resins. In general, the kinetic models such as pseudo-first-order and pseudo-second-order models are applied to predict the sorption kinetics of rutin adsorption ${ }^{36,37}$ (Figure 6). The pseudo-first-order model assumes that the physical adsorption process (the interactions between the adsorbent and the adsorbate are van der Waals force, the hydrogen bond force, etc.) is the rate-controlling step, whereas the pseudo-second-
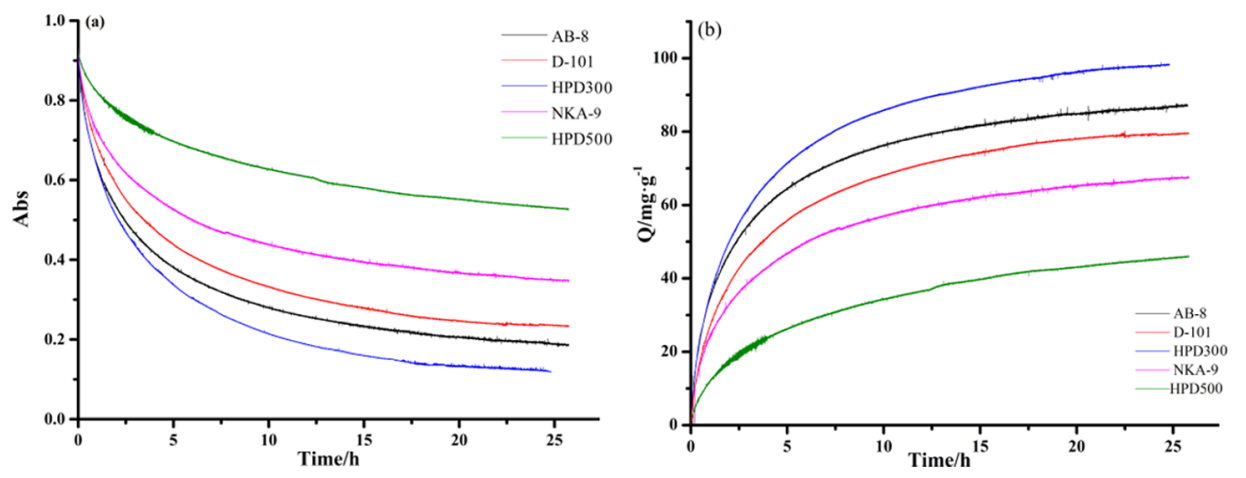

Figure 3. (a) Real-time change of absorbance with time and (b) adsorption kinetic curves of rutin on different MARs in aqueous solution, $C_{0}=60$ $\mathrm{mg} / \mathrm{L}, \mathrm{T}=20^{\circ} \mathrm{C}$, measured at $353 \mathrm{~nm}$. 

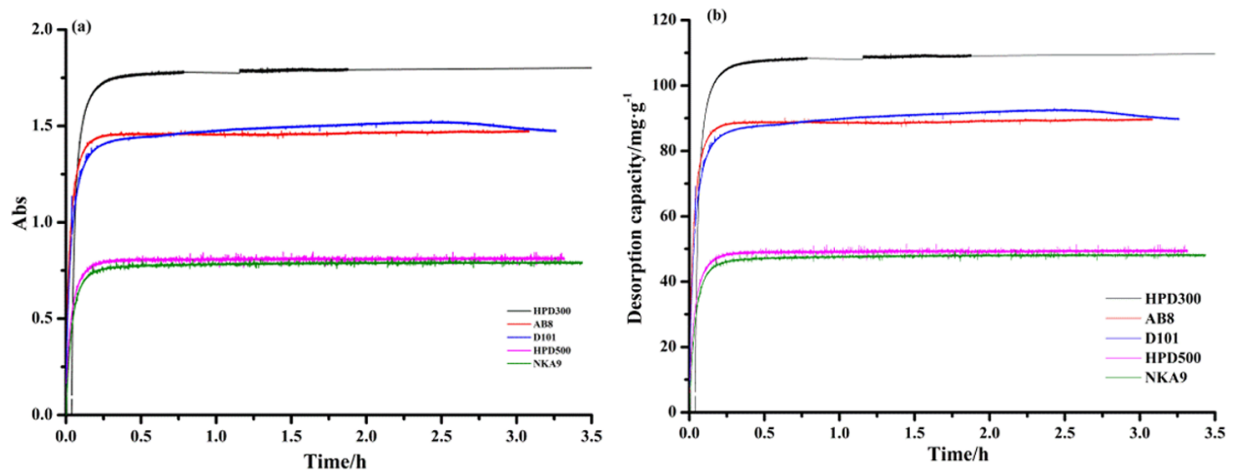

Figure 5. (a) Absorbance curves of rutin on different MARs during the desorption process, (b) desorption curves of rutin on different MARs (the desorption solution was ethanol aqueous solution; $T=30^{\circ} \mathrm{C}$ ).
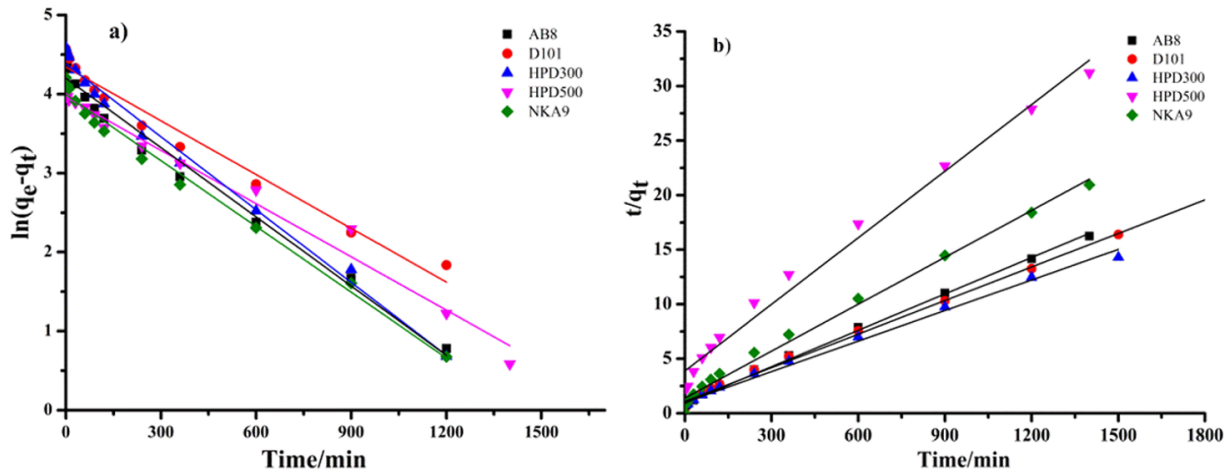

Figure 6. Fitting results of rutin on resins for adsorption kinetic models, (a) pseudo-first-order model and (b) pseudo-second-order model: $C_{0}=60$ $\mathrm{mg} / \mathrm{L} ; t=26 \mathrm{~h}$.

Table 1. Parameters in the Pseudo-First-Order and Pseudo-Second-Order Rate Equations for Rutin on Resins ${ }^{a}$

\begin{tabular}{|c|c|c|c|c|c|c|c|}
\hline \multirow[b]{2}{*}{ resins } & \multirow[b]{2}{*}{${ }^{\mathrm{a}} \mathrm{Q}_{\mathrm{e}, \exp }\left(\mathrm{mg} \cdot \mathrm{g}^{-1}\right)$} & \multicolumn{3}{|c|}{ pseudo-first-order } & \multicolumn{3}{|c|}{ pseudo-second-order } \\
\hline & & ${ }^{\mathrm{b}} \mathrm{Q}_{\mathrm{e}, \mathrm{cal}}\left(\mathrm{mg} \cdot \mathrm{g}^{-1}\right)$ & $k_{1} \times 10^{-3}\left(\mathrm{~min}^{-1}\right)$ & $R^{2}$ & $Q_{e, \text { cal }}\left(\mathrm{mg} \cdot \mathrm{g}^{-1}\right)$ & $k_{2} \times 10^{-4}\left(\mathrm{~g} \cdot \mathrm{mg}^{-1} \cdot \mathrm{min}^{-1}\right)$ & $R^{2}$ \\
\hline $\mathrm{AB}-8$ & 86.92 & 72.39 & 33.71 & 0.9797 & 90.91 & 1.276 & 0.9951 \\
\hline HPD300 & 98.30 & 89.47 & 34.56 & 0.9872 & 104.2 & 0.8517 & 0.9903 \\
\hline HPD500 & 55.47 & 51.24 & 23.18 & 0.9814 & 56.27 & 0.7442 & 0.9893 \\
\hline NKA-9 & 67.19 & 54.06 & 27.75 & 0.9867 & 70.42 & 1.377 & 0.9917 \\
\hline D101 & 94.83 & 73.18 & 20.13 & 0.9683 & 100.0 & 0.9099 & 0.9940 \\
\hline
\end{tabular}

${ }^{a_{\mathrm{a}}} Q_{e, \text { exp }}$ and ${ }^{\mathrm{b}} Q_{e, \text { cal }}$, apparent and calculated equilibrium adsorption capacity, respectively; $k_{1}$, pseudo-first-order rate constant; $k_{2}$, pseudo-secondorder rate constant; $R^{2}$, correlation coefficient.

order model assumes that the chemisorption of the adsorbate onto the active adsorption sites (electron sharing and electron exchange between the adsorbent and the adsorbate) is the ratecontrolling step, and the linear form of the two models are described by the following equations

$$
\begin{aligned}
& \text { pseudo-first-order: } \ln \left(q_{\mathrm{e}}-q_{t}\right)=\ln q_{\mathrm{e}}-k_{1} t \\
& \text { pseudo-second-order: } \frac{t}{q_{t}}=\frac{1}{k_{2} q_{\mathrm{e}}{ }^{2}}+\frac{t}{q_{\mathrm{e}}}
\end{aligned}
$$

where $k_{1}$ and $k_{2}$ are pseudo-first-order rate constant $\left(\mathrm{min}^{-1}\right)$ and pseudo-second-order rate constant $\left(\mathrm{g} \cdot \mathrm{mg}^{-1} \cdot \mathrm{min}^{-1}\right), q_{t}$ and $q_{\mathrm{e}}\left(\mathrm{mg} \cdot \mathrm{g}^{-1}\right)$ are the adsorption amount at time $t$ and equilibrium, respectively.

Table 1 lists the fitting parameters, such as adsorption capacities (Q), adsorption rate constant $(k)$, and the correlation coefficients $\left(R^{2}\right)$ of the adsorption kinetic curves. The correlation coefficients obtained from the pseudo-firstorder kinetic model are all smaller than 0.99. However, the correlation coefficients from the pseudo-second-order kinetic model are in between 0.99 and 1 except for that of HPD500, and it is better than that of pseudo-first-order kinetic model. The theoretical value of $q_{\mathrm{e}}$ from the pseudo-first-order kinetic model is significantly lower than that of the experimental data. Therefore, the pseudo-second-order model is more suitable for this adsorption. In addition, the initial adsorption rate $v_{0}$ of the five resins described by $v_{0}=k_{2} q_{\mathrm{e}}{ }^{2}$ was calculated from the pseudo-second-order kinetic model and was 1.055, 1.052, $0.2907,0.7042$, and $0.9099 \mathrm{mg} \cdot \mathrm{g}^{-1} \cdot \mathrm{min}^{-1}$, respectively. Among them, AB- 8 showed the highest initial adsorption rate for rutin and this result was in good agreement with data on the reported work. ${ }^{38}$

\section{CONCLUSIONS}

To conclude, a new method based on the self-made FOS system was developed for in situ, real-time measurement of LPA by making good use of FOS and membrane separation. It opened a new path to circumvent the absence of method for 
fast (few minutes) adsorption and lack of automatic measurement. FOS allowed the system to collect several adsorption data in every second and operate several days continuously. The system can eliminate human error and operation fatigue thanks to in situ measurement and thereby ensured high accuracy and high reproducibility owing to all-in-one pot, automatic process with strictly controlled condition. In order to realize the full scientific and commercial opportunities enabled by this technique, many more studies remain to be performed, and including application of the system for the measurement of adsorption thermodynamics, alternative adsorption measurement vessels, and validation of the system in wide variety of adsorptions and in real samples (such as plant extractions) will be investigated. Furthermore, some new insights into the LPA and adsorption will be gained.

\section{EXPERIMENTAL SECTION}

4.1. Materials. Five macroporous resins (MARs) including AB-8, D101, HPD300, HPD500, HPD600, and NKA-9 were purchased from Cangzhou Bon Adsorber Technology Co., Ltd (Hebei, China). Rutin was obtained from (Nanjing, China) with a purity higher than $98 \%$. Analytical grade methanol and ethanol were purchased from Sinopharm Chemical Reagent (Shanghai, China) and ultrapure water was obtained from a water purification system (Chengdu, China).

4.2. Instrumentation. The components for in situ fiberoptic detection system for LPA and their photographs are shown in Figures 7 and S6, respectively. The system consists of

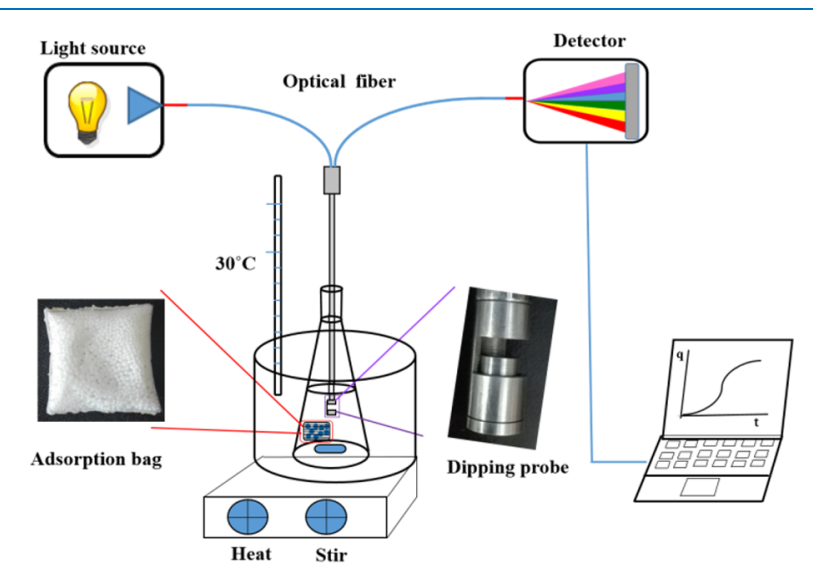

Figure 7. Schematic illustration of the in situ real-time measurement system of liquid phase adsorption.

a stable and continuous source of spectrum light (195-644 $\mathrm{nm}$ ) which was provided by a DH-2000 deuterium tungsten halogen source. An HR2000 charge-coupled device spectrophotometer was connected to the light source by a fiber-optic dipping probe (all parts from Ocean Optics, Dunedin, Florida, USA). The probe includes an Al-coated mirror, an illumination fiber, and all mounted in stainless steel cylinders. Adsorption vessel mainly contains a conical flask, magnetic stirrer, adsorption bag, and fiber-optic probe.

4.3. Activation of MARs. Five MARs' physical properties are listed in Table S1. Certain amounts of MARs were sealed into a 100 -mesh nylon porous bag $(2 \times 2 \mathrm{~cm})$ and placed into a conical flask with a cover, and then, the resins were first soaked in ethanol for $46 \mathrm{~h}$ and then washed (the white turbidity) with ultrapure water till no residual ethanol could be detected. ${ }^{19}$ After being dried naturally, the activated and purified MARs were obtained in the form of adsorption bag.

4.4. Rutin Standard Solutions. A stock solution of rutin was prepared by dissolving $0.0600 \mathrm{~g}$ rutin with ultrapure water at $80{ }^{\circ} \mathrm{C}$ and then transferred into a $1000 \mathrm{~mL}$ volumetric flask after cooling down to room temperature. ${ }^{19}$ Different portions were accurately pipetted from standard solution into several $100 \mathrm{~mL}$ volumetric flasks and diluted with water to obtain a series of standard solutions in the range of $0.3-60 \mathrm{mg} / \mathrm{L}$, respectively.

4.5. System Configuration and Standard Curve. The rutin solution concentration was detected by the in situ fiberoptic spectrophotometer at $353 \mathrm{~nm}$ according to the procedures as follows: (1) deuterium light source was turned on for at least $30 \mathrm{~min}$ to pre-warm, and then, the spectra suite software was opened, the integration time was adjusted to 18 $\mathrm{ms}$, and the smoothing width was set to 5. (2) An optical probe with a $5 \mathrm{~mm}$ light path was selected for the measurement and dipped into blank solvent (water), the signal intensity level was adjusted to 3500 counts at the corresponding detection wavelength $(353 \mathrm{~nm})$, and reference and dark spectra were stored. (3) The probe was dipped into the vessels that contain $3 \mathrm{~mL}$ of rutin solution of different concentrations, respectively. Then, the absorbance of each rutin solution was obtained. The same system configuration was used for the rest of the experiment.

4.6. In Situ Adsorption and Desorption Kinetics. 4.6.1. Adsorption Test. Before each adsorption experiment, the corresponding spectral parameters were adjusted to the same as subsection 4.5. After configuring the parameters, the probe immersed in the adsorption vessel, which was filled with 200 $\mathrm{mL}$ of the rutin solution at $20^{\circ} \mathrm{C}$. Then, the time sequence test function was activated with setting the detection and reference wavelengths to 353 and $453 \mathrm{~nm}$, respectively. After that, $0.1 \mathrm{~g}$ of pretreated resin adsorption bag was put into the vessel with magnetic stirring at $310 \mathrm{rpm}$. The absorbance of the rutin solution was recorded at a frequency of $4 / \mathrm{s}$. If the absorption change is not more than 0.002 within $2 \mathrm{~h}$ (slope $\Delta A / \Delta t<$ 0.001 ), it is decided that the adsorption reaches apparent equilibrium stage. In the end, the light source was switched off and the data were stored. With these procedures, the timeabsorbance curve at $353 \mathrm{~nm}$ (Figure S7) was obtained according to the confirmed parameters. The rutin concentration in the adsorption process was determined with a fiberoptic spectrometer at different time intervals until equilibrium. The adsorption capacity of the rutin was calculated by using eq 3

$$
Q_{t}=\left(C_{0}-C_{t}\right) \times V / m
$$

where $Q_{t}$ stands for the adsorption capacity of the MARs at the time $t(\mathrm{mg} / \mathrm{g}), C_{0}$ for initial rutin concentration and $C_{t}$ stands for the rutin concentration $(\mathrm{mg} / \mathrm{L})$ at time $t, V$ is the volume of the adsorption solution (L), and $m$ indicates the weight of the MARs (g).

4.6.2. Desorption Test. After the adsorption reached equilibrium, the adsorption bags were removed and washed twice with ultrapure water. The signal intensity level was adjusted to 3500 counts in the blank solvent, which was ethanol solution ( $\mathrm{v} / \mathrm{v}, 70 \%$ ethanol/water). Then, the adsorption bag was put into the flask, which contains 100 $\mathrm{mL}$ of ethanol solution at $30{ }^{\circ} \mathrm{C}$ with magnetic stirring at 310 $\mathrm{rpm}$. The absorbance of rutin at every $250 \mathrm{~ms}$ was recorded until desorption equilibrium by in situ fiber-optic spectrom- 
etry. Then, the desorption capacity of each rutin on the resin was calculated according to eq 4

$$
D_{\mathrm{c}}=\left(C_{\mathrm{d}} \times V_{\mathrm{d}}\right) / m, \quad D=D_{\mathrm{c}} / Q_{\mathrm{e}}
$$

where $D_{c}$ and $D$ denote the desorption capacity and desorption ratio (\%), respectively, $C_{\mathrm{d}}$ is the concentration of the rutin in the final desorption solution $(\mathrm{mg} / \mathrm{L}), V_{\mathrm{d}}$ represents the volume of the desorption solution (L), $Q_{e}$ stands for the adsorption capacity of the MARs $(\mathrm{mg} / \mathrm{g})$, and $m$ is the weight of the MARs (g).

\section{ASSOCIATED CONTENT}

\section{S Supporting Information}

The Supporting Information is available free of charge on the ACS Publications website at DOI: 10.1021/acsomega.8b01059.

Chemical structure of rutin; effect of nylon net on adsorption; repeated adsorption measurement of rutin on $\mathrm{AB}-8$; RSD of rutin adsorption on $\mathrm{AB}-8$ for repeated measurement; comparison of the in situ system with conventional liquid phase measurement method; photographic image of adsorption measurement system; realtime change of absorbance with time during adsorption of rutin on $\mathrm{AB}-8$; physical properties of five MARs; and statistical comparison of the two methods (PDF)

\section{AUTHOR INFORMATION}

\section{Corresponding Author}

*E-mail: turghunm@sina.com. Phone: +86 9918582564. Fax: +869918582809.

\section{ORCID}

Turghun Muhammad: 0000-0001-5562-9365

Lingxin Chen: 0000-0002-3764-3515

\section{Author Contributions}

T.M. and F.G. conceived the idea and designed the experiments. F.G. and P.P. performed the experiments. T.M., F.G., and M.B. analyzed the data. T.M., F.G., and L.C. wrote the manuscript. T.M. and L.C. supervised the research.

\section{Notes}

The authors declare no competing financial interest.

\section{ACKNOWLEDGMENTS}

This work was supported by the National Natural Science Foundation of China (21365020 and 21565025). We thank Jinhua $\mathrm{Li}$ for the revision of the manuscript.

\section{REFERENCES}

(1) Huang, W. Q.; Li, C. X. Adsorption Separation Material; Chemical Industry Press: Beijing, 2005.

(2) Li, G. X. Adsorption Science; Chemical Industry Press: Beijing, 2006

(3) Thomas, R. C.; Sun, L.; Crooks, R. M. Real-Time Measurements of the Gas-Phase Adsorption of n-Alkylthiol Mono- and Multilayers on Gold. Langmuir 1991, 7, 620-622.

(4) Takenouchi, M.; Kudoh, S.; Miyajima, K.; Mafuné, F. Adsorption and Desorption of Hydrogen by Gas-Phase Palladium Clusters Revealed by In Situ Thermal Desorption Spectroscopy. J. Phys. Chem. A 2015, 119, 6766-6772.

(5) Ali, I.; Gupta, V. K. Advances in water treatment by adsorption technology. Nat. Protoc. 2007, 1, 2661-2667.
(6) Huang, Y.; Li, C.; Lin, Z. EDTA-Induced Self-Assembly of 3D Graphene and Its Superior Adsorption Ability for Paraquat Using a Teabag. ACS Appl. Mater. Interfaces 2014, 6, 19766-19773.

(7) Leyton, A.; Vergara-Salinas, J. R.; Pérez-Correa, J. R.; Lienqueo, M. E. Purification of phlorotannins from Macrocystis pyrifera using macroporous resins. Food Chem. 2017, 237, 312-319.

(8) Kim, T.-S.; Song, H. J.; Dar, M. A.; Lee, H.-J.; Kim, D.-W. Fast adsorption kinetics of highly dispersed ultrafine nickel/carbon nanoparticles for organic dye removal. Appl. Surf. Sci. 2018, 439, 364-370.

(9) Khan, M. A.; Wallace, W. T.; Islam, S. Z.; Nagpure, S.; Strzalka, J.; Littleton, J. M.; Rankin, S. E.; Knutson, B. L. Adsorption and Recovery of Polyphenolic Flavonoids Using TiO2-Functionalized Mesoporous Silica Nanoparticles. ACS Appl. Mater. Interfaces 2017, 9, 32114-32125.

(10) Zhao, Z. G. Application Principle of Adsorption; Chemical Industry Press: Beijing, 2005.

(11) Pan, S.-D.; Ye, M.-J.; Gao, G.-S.; He, Q.; Wang, L.; Chen, X.H.; Qiu, Q.-L.; Jin, M.-C. Synthesis of a monodisperse well-defined core-shell magnetic molecularly-imprinted polymer prior to LC-MS/ MS for fast and sensitive determination of mycotoxin residues in rice. Anal. Methods 2017, 9, 5281-5292.

(12) Sun, X. D.; Li, S. H.; Ming Dong, X. Y.; Wang, L.-j.; Gu, W.-b.; Wang, W.-m.; Yang, Z.-y.; Wang, Y.; Zhu, J. H. Liquid adsorption and catalytic degradation of 4-methylnitrosamino-1-3-pyridyl-1-butanone (NNK) by zeolite. Microporous Mesoporous Mater. 2017, 243, 39-46.

(13) Han, L. M.; Wang, S. Q.; Ju, H. Y.; Yan, F. GC-MS analysis on the kinds of soybean root exudates extracted with adsorption resin. Soybean Sci. 2003, 22, 301-305.

(14) Lian, G.; Zhang, X.; Si, H.; Wang, J.; Cui, D.; Wang, Q. Boron Nitride Ultrathin Fibrous Nanonets: One-Step Synthesis and Applications for Ultrafast Adsorption for Water Treatment and Selective Filtration of Nanoparticles. ACS Appl. Mater. Interfaces 2013, 5, 12773-12778.

(15) Jiang, J.-Q.; Yang, C.-X.; Yan, X.-P. Zeolitic Imidazolate Framework-8 for Fast Adsorption and Removal of Benzotriazoles from Aqueous Solution. ACS Appl. Mater. Interfaces 2013, 5, 98379842.

(16) Liu, J. B. Studies on the Separation and Purification of Flavonoids with Macroporous Adsorption Resins. Master Dissertation, Zhejiang University, Hangzhou, 2012.

(17) Di Bello, M. P.; Mergola, L.; Scorrano, S.; Del Sole, R. Towards a new strategy of a chitosan-based molecularly imprinted membrane for removal of 4-nitrophenol in real water samples. Polym. Int. 2017, 66, 1055-1063.

(18) MacKenzie, L. A. In situ passive solid-phase adsorption of micro-algal biotoxins as a monitoring tool. Curr. Opin. Biotechnol. 2010, 21, 326-331.

(19) Chen, Z.; Zhang, A.; Li, J.; Dong, F.; Di, D.; Wu, Y. Study on the Adsorption Feature of Rutin Aqueous Solution on Macroporous Adsorption Resins. J. Phys. Chem. B 2010, 114, 4841-4853.

(20) Wang, X.-d.; Wolfbeis, O. S. Fiber-Optic Chemical Sensors and Biosensors (2013-2015). Anal. Chem. 2016, 88, 203-227.

(21) Li, Q.; Qiu, T.; Hao, H.; Zhou, H.; Wang, T.; Zhang, Y.; Li, X.; Huang, G.; Cheng, J. Rapid and on-site analysis of illegal drugs on the nano-microscale using a deep ultraviolet-visible reflected optical fiber sensor. Analyst 2012, 137, 1596-1603.

(22) Wang, X.-D.; Wolfbeis, O. S. Fiber-Optic Chemical Sensors and Biosensors (2008-2012). Anal. Chem. 2013, 85, 487.

(23) Muhammad, T.; Wang, J.; Li-wan, M.; Chen, J. Monitoring Dissolution Rate of Amiodarone Tablets by a Multiple Fiber-Optic Sensor System. Dissolution Technol. 2008, 15, 22-27.

(24) Nie, K.; Li, L.; Li, X.; Geng, D.; Zhang, Q.; Tuo, M.; Xu, P.; Chen, J. In Situ Fiber-Optic Dissolution Assisted by a Mathematical Separation Model of Dynamic Three-Wavelength K-Ratio Spectrophotometry. Dissolution Technol. 2010, 17, 15-18.

(25) Seif, S.; Graef, F.; Gordon, S.; Windbergs, M. Monitoring Drug Release from Electrospun Fibers Using an In Situ Fiber-Optic System. Dissolution Technol. 2016, 23, 6-11. 
(26) Josefson, M.; Johansson, E.; Torstensson, A. Optical fiber spectrometry in turbid solutions by multivariate calibration applied to tablet dissolution testing. Anal. Chem. 1988, 60, 2666-2671.

(27) Mongstad, T.; Platzer-Björkman, C.; Maehlen, J. P.; Mooij, L. P. A.; Pivak, Y.; Dam, B.; Marstein, E. S.; Hauback, B. C.; Karazhanov, S. Z. A new thin film photochromic material: Oxygen-containing yttrium hydride. Sol. Energy Mater. Sol. Cells 2011, 95, 3596-3599.

(28) Wang, R.; Yang, G.; Zhang, J.; Li, Q.; Fu, R.; Ye, J.; Wang, T.; Lu, Y.; Zhou, H.; Huang, G. Rapid, on-site detection of residual explosives based on a lab-in-a-capillary and UV fiber sensor. Anal. Methods 2014, 6, 9628-9633.

(29) Zhang, H.-B.; Xiao, W.-J.; Zhao, D.-S.; Liu, Y.; Hu, S.-C.; Chen, C.-L.; Xu, P.-P.; Li, X.-X.; Chen, J. Fiber-optic in situ analysis of the catalytic kinetics of the alliin/alliinase system. J. Mol. Catal. B: Enzym. 2014, 106, 111-116.

(30) Muhammad, T.; Yimit, O.; Turahun, Y.; Muhammad, K.; Uludağ, Y.; Zhao, Z. On-line determination of 4-nitrophenol by combining molecularly imprinted solid-phase extraction and fiberoptic spectrophotometry. J. Sep. Sci. 2014, 37, 1873-1879.

(31) Zhao, Y. Fiber Optic Sensing Principle and Application Technology; Tsinghua University Press: Beijing, 2007.

(32) Meng, L.; Liu, R.; Sun, A.; Wu, S. J.; Liu, N. Separation and Purification of Rutin and Acaciin from the Chinese Medicinal Herb Herba Cirsii by Combination of Macroporous Absorption Resin and High-Speed Counter-Current Chromatography. J. Chromatogr. Sci. 2009, 47, 329-332.

(33) Zhao, Z.; Dong, L.; Wu, Y.; Lin, F. Preliminary separation and purification of rutin and quercetin from Euonymus alatus (Thunb.) Siebold extracts by macroporous resins. Food Bioprod. Process. 2011, 89, 266-272.

(34) Sinwat, W.; Sappat, A.; Tuantranont, A.; Laiwattanapaisal, W. On-site determination of microalbuminuria based on Particle Enhanced Turbidimetric-Inhibition Immunoassay (PETINIA) by portable fiber-optic spectrometer. J. Chem. Pharmaceut. Res. 2012, 4, 2879-2887.

(35) Saita, T.; Fujito, H.; Mori, M. Development of an EnzymeLinked Immunosorbent Assay for the Quantification of Amiodarone. Biol. Pharm. Bull. 2002, 25, 954-958.

(36) Molavi, H.; Hakimian, A.; Shojaei, A.; Raeiszadeh, M. Selective dye adsorption by highly water stable metal-organic framework: long term stability analysis in aqueous media. Appl. Surf. Sci. 2018, 445, 424-436.

(37) Lou, S.; Liu, Y. F.; Bai, Q. Q.; Di, D. L. Adsorption Mechanism of Macroporous Adsorption Resins. Prog. Chem. 2012, 24, 14271436.

(38) Li, Y.; Liu, J.; Cao, R.; Deng, S.; Lu, X. Adsorption of Myricetrin, Puerarin, Naringin, Rutin, and Neohesperidin Dihydrochalcone Flavonoids on Macroporous Resins. J. Chem. Eng. Data 2013, 58, 2527-2537. 